\title{
The Slewing Mirror Telescope of UFFO-pathfinder: first performance report in space
}

G. Gaikov ${ }^{1 *}$, S. Jeong ${ }^{1},{ }^{2}$ V.G. Agaradahalli ${ }^{1} ;$ I.H. Park ${ }^{1 \S}$ A.M. Amelushkin ${ }^{3}$, V.O.

Barinova $^{3}$, A.V. Bogomolov ${ }^{3}$, V.V. Bogomolov ${ }^{3}$, C. Budtz-Jørgensen ${ }^{4}$, A.J.

Castro-Tirado ${ }^{2}{ }^{6}$, P. Chen ${ }^{8},{ }^{5}$, N.L. Dzhioeva ${ }^{3}$, E.S. Gorbovskoy ${ }^{3}$, A.F. lyudin ${ }^{3}$, V.V.

Kalegaev $^{3}$, P.S. Kasarjan ${ }^{3}$, H.M. Jeong ${ }^{1}$, J.E. Kim ${ }^{1}$, M.B. Kim ${ }^{1}$, S.W. Kim ${ }^{7}$, V.G. Kornilov ${ }^{3}$, E.A. Kuznetsova ${ }^{3}$, J. Lee ${ }^{1}$, V.M. Lipunov ${ }^{3}$, I.N. Myagkova ${ }^{3}$, J.W. Nam ${ }^{8}{ }^{5}$, M.I. Panasyuk ${ }^{3}$, M.I. Panchenko ${ }^{3}$, V. Petrov ${ }^{3}$, A.V. Prokhorov ${ }^{3}$, V. Reglero ${ }^{9}$, A.N. Shustova ${ }^{3}$, S. Svertilov ${ }^{3}$, N.V Tyurina ${ }^{3}$, I. Yashin ${ }^{3}$

${ }^{1}$ Department of Physics, Sungkyunkwan University, 2066, Seobu-ro, Jangan-gu, Suwon,

Gyeonggi-do, 16419, Korea

${ }^{2}$ Instituto de Astrofisica de Andalucia ZAA-CSIC,P. O. Box 03004, E-18080 Granada, Spain

${ }^{3}$ Skobeltsyn Institute of Nuclear Physics of Lomonosov, Moscow State University, Leninskie

Gory, Moscow, 119234, Russia

${ }^{4}$ National Space Institute, Technical University of Denmark, 2800 Kgs. Lyngby, Denmark

5 Leung Center for Cosmology and Particle Astrophysics, National Taiwan University, 1

Roosevelt Road, Taipei, 10617, Taiwan

${ }^{6}$ Departamento de Ingeniería de Sistemas y Automática, Universidad de Málaga, Spain

${ }^{7}$ Department of Astronomy, Yonsei University, 134 Shinchon-dong, Seoul 120-749, Korea

${ }^{8}$ Department of Physics, National Taiwan University, 1 Roosevelt Road, Taipei, 10617, Taiwan

${ }^{9}$ GACE, Edif. de Centros de Investigacion, Universidad de Valencia, Burjassot, E-46100

Valencia, Spain

E-mail: ilpark@skku.edu

The Slewing Mirror Telescope (SMT) is the first orbital instrument, using motorized mirror for observations of the space, designed to shed light on first seconds of Gamma-Ray Bursts (GRBs) prompt UV/optical emission. The SMT is main component of the Ultra-Fast Flash Observatory (UFFO). Once GRB is detected by UFFO X-ray coded mask camera, SMT turns its motorized mirror within seconds to observe optical and UV emission of GRB. After several years of delay on its launch, UFFO was finally launched in 28 April 2016. Launch took place onboard Lomonosov satellite from new Russian kosmodrom Vostochny. Several space tests have been performed during that period, which proved the concept of SMT and gave precise values of SMT sensitivity and resolution. For the first time in the space observations, motorized mirror system is able to trace objects despite the drift of telescope FOV due to satellite orbital movement.

35th International Cosmic Ray Conference - ICRC2017

10-20 July, 2017

Bexco, Busan, Korea

\footnotetext{
*Speaker.

${ }^{\dagger}$ Co-first author

$¥$ On leave of Birla Institute of Technology and Science, India

$\S_{\text {Corresponding author }}$
} 


\section{Introduction}

One of most important astrophysical objects for emerging multi-messenger astronomy are Gamma-Ray Bursts (GRBs) (Willingale and Meszaros, 2017). Observation of Gamma-Ray Bursts (GRBs) in different wavelengths accompanied with gravitational waves (Connaughton et al., 2016) are of main interest in space science. In 2004, NASA's SWIFT (Gehrels et al., 2004) space observatory provided first information of GRB in a variety of optical / UV wavelengths. simultaneously measuring x-ray and UV/optical signals in a few different filters. Principle of SWIFT working is reorientation of whole spacecraft towards GRB destination, resulting in $\sim 100 \mathrm{sec}$ reaction time.

A new project, Ultra-Fast-Flash observatory (UFFO-pathfinder Park et al. (2012)), launched in April 2016 onboard Lomonosov (Park et al., 2017) satellite, is based on a concept of Slewing Mirror Telescope (SMT) (Jeong et al., 2013). SMT employs a rapidly moving motorized mirror (see Fig. 1) to redirect the optical path of telescope towards GRB instead of moving the whole spacecraft. Therefore, UFFO-pathfinder has one second level reaction time and is able to study early time domain of GRBs. Since its launch, we have confirmed the concept of slewing mirror telescope, which is key idea of UFFO-pathfinder.

SMT consists of $100 \mathrm{~mm}$ Ritchey-Chrétien telescope detailed in Jeong et al. (2013); slewing mirror system and readout electronics (Kim et al., 2013). SMT is built to cover half-coded FoV of UFFO Burst Alert and Trigger Telescope (UBAT - Jeong et al. (2017)), i.e. $\pm 35^{\circ}$ using slewing mirror. Data acquisition strategy of SMT is 1-bit $20 \mathrm{~ms}$ exposure frames, with a $20 \mathrm{~ms}$ gap time between frames.

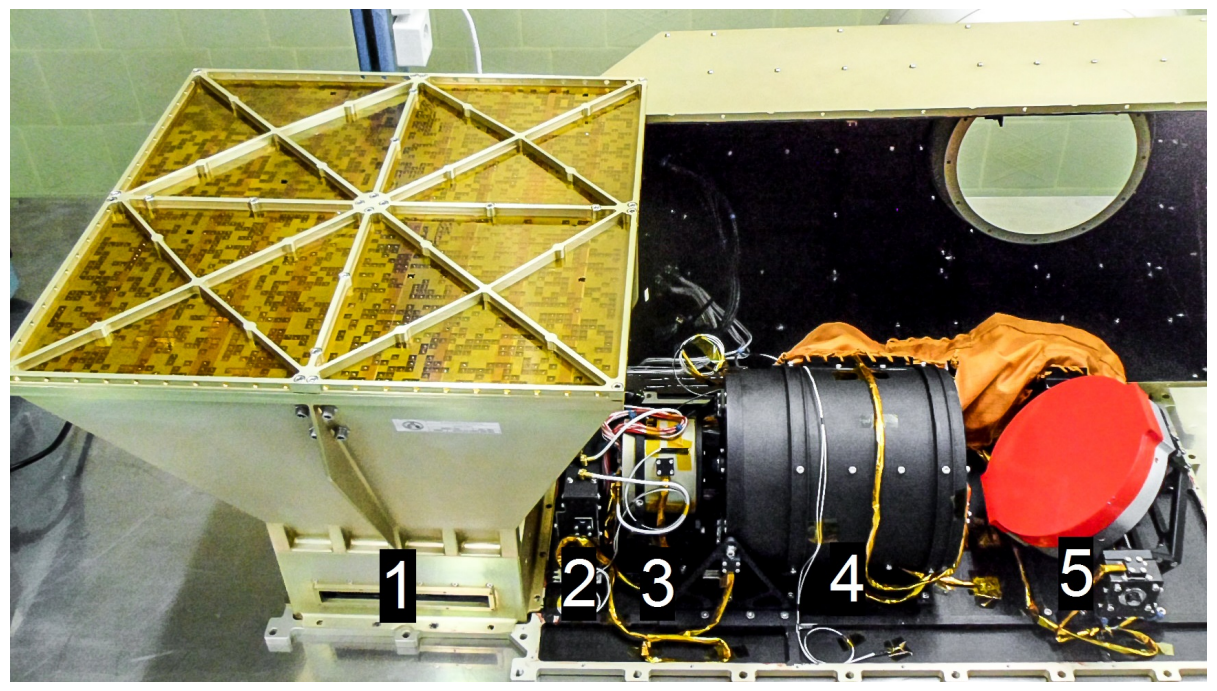

Figure 1: Ultra-Fast Flash Observatory Pathfinder. (1) - UFFO-pathfinder Burst Alert and Trigger Telescope (UBAT) - X-ray coded musk camera, used for trigger and localization of GRB; Slewing Mirror Telescope: (2) - Readout electronics; (3) - Intensified charge-coupled device detector (ICCD); (4) - $10 \mathrm{~cm}$ RC Telescope; (5) - Slewing mirror system (the mirror is under a red protection cover before launch).

\section{Performance in space}

Being launched in 28 April 2016, UFFO-pathfinder was first turned on in June 2016. After 
that, we have performed series of tests onboard. In the following we show STM's performance in the space and proofs of work of the slewing mirror concept.

\subsection{Observed issues}

First several tests, taken place in June 2016, brought no data from UFFO-pathfinder, despite the fact that there were triggers produced by UBAT. At default settings, data is not saved unless mirror reaches correct trigger position, so motor system was suspected to be the possible reason of failure.

We performed a detailed study of available space logs (i.e., current consumption), which showed that a problem happened with mirror position reading system: commercial decoder board (VK10S08B0AN, Gurley Precision Instrument) started to work incorrectly. Decoder board reads motor A and motor B position values using two sensors, writes each motor position as a 16-bit value and transports them to the main SMT FPGA. The decoder board started to send wrong position values (see Fig. 2) for the most of possible positions of motor A. Two plots are showing results of mirror position reading system tests on ground and in space; horizontal axis corresponds to real motors position; vertical axis is 16-bit value, send by decoder board to main SMT FPGA. In normal case, this dependence must be linear, as we see on ground data. However, space data for motor A has completely different pattern after launch. We suspect damage of decoder FPGA logic by cosmic radiation environment as a possible reason of this hardware fail.
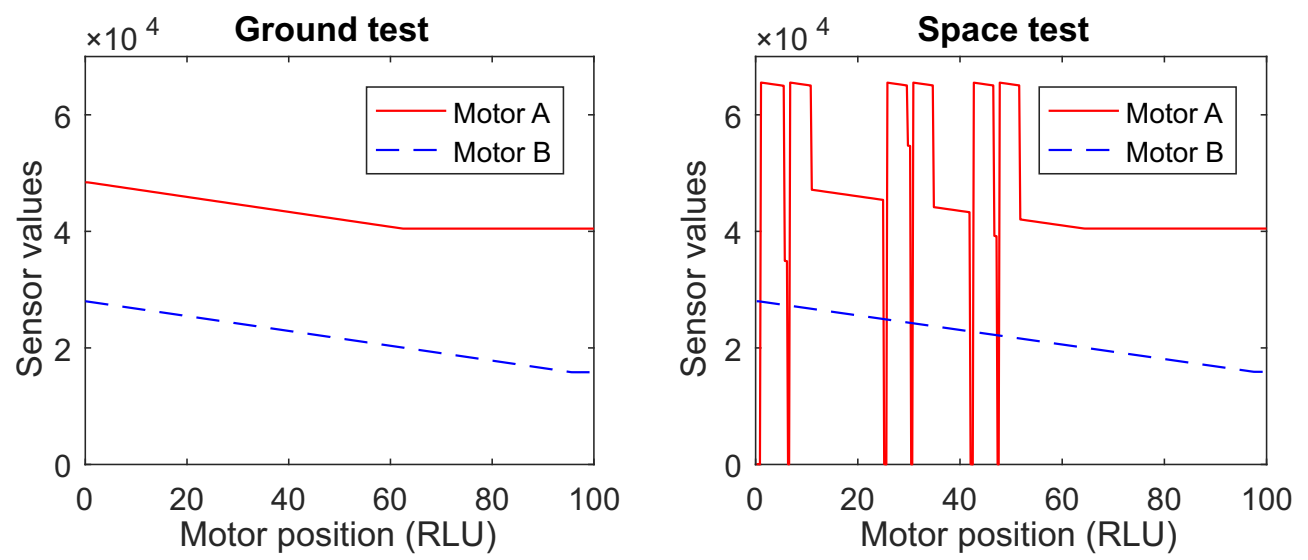

Figure 2: Results of mirror position reading system tests on ground and in space. Right plot demonstrates breakage of motor A position reading system after launch: at some positions of motor A, feedback system gives out completely wrong angle values.

With the failure happened, main SMT board was getting wrong data about current mirror position, even though motors were working correctly.

Possible problems with mirror position reading system were foreseen before the flight, and some precautions were taken. We implemented several options in the code, which allows motor to rotate in "blind" way - without feedback. Accuracy of this mode is order of 2 ', which is lower, than with feedback. Another disadvantage is that without position reading system we cannot be sure, that SMT is looking in the proper direction. Astrometry becomes the only way left to check coordinates of SMT FoV. 
Table 1: Slewing mirror performance: response time to the trigger and time of stable FoV (maximal time, for which a target could be observed by telescope

\begin{tabular}{|c|c|c|c|c|}
\hline \multirow[b]{2}{*}{ Date } & \multicolumn{2}{|c|}{ Target position 1} & \multirow{2}{*}{$\begin{array}{l}\text { Response } \\
\text { time, sec }\end{array}$} & \multirow{2}{*}{$\begin{array}{l}\text { Tracking } \\
\text { time, } \text { sec }^{2}\end{array}$} \\
\hline & $\mathrm{X}$, degree & Y, degree & & \\
\hline Sep 26 & 8.58 & -25.67 & 1.36 & 148 \\
\hline Sep 29 & 13.67 & -19.67 & 1.72 & 88 \\
\hline Oct 4 & 13.75 & -19.18 & 1.08 & 40 \\
\hline Oct 20 & 10.45 & 1.28 & 1.47 & 170 \\
\hline Nov 10 & -4.55 & 5.92 & 1.04 & 191 \\
\hline
\end{tabular}

${ }^{1}$ In the UFFO-pathfinder own system of coordinates.

${ }^{2}$ Is estimated ratio of FoV size to remaining drift speed.

\subsection{Response time}

Main purpose of UFFO-pathfinder is studying of the first seconds of GRB prompt optical and UV emissions. Therefore, response time of SMT after the UBAT trigger is one of the main UFFOpathfinder parameters. Tab. 1 contains the results of five SMT response time measurement tests for given triggers of UBAT. In general, response time is smaller, when target position is close to mirror initial position, like November 10 data.

Despite the mirror position reading system problems, described in Sec. 2.1, SMT still has $1.4 \pm 0.3$ seconds response time, and thus fully meets the scientific goal of UFFO-pathfinder.

\subsection{Tracking performance}

SMT is the first space telescope using slewing mirror for tracking astrophysical objects. FoV of SMT is 17'; drift of FoV speed is $4^{\prime} / \mathrm{sec}$ (this is speed of orbital movement of Lomonosov) - thus, without drift correction, FoV should completely change after 4 seconds. SMT rotates the mirror, keeping FoV always in same position.

Correction of FoV is made every second. Fig. 3 shows how FoV is drifting during one second (frames 1-15) and how this drift is compensated by mirror system (frames 16-20). Each next 1bit frame is shifted from previous, thus, combining them without software adjustment makes final image blurred. At the frame 20, bright star returns to almost same position, as at first frame. The random mistake of FoV correction is around 1' (with mirror position reading system it could be more precise - see Sec. 2.1). This imperfection of motor system work is overcomed by data combining software on ground, by finding 2D cross correlation function between images with slightly different FoV.

Despite small errors in mirror drift correction, SMT is able to track astrophysical objects for $150 \mathrm{sec}$ - see Tab. 1. Thus, slewing mirror system fully meets its scientific goal - SMT is able to observe first 100 seconds of GRB.

\subsection{Imaging performance}

Point spread function of SMT has $4.2^{\prime \prime} \sigma$, as was checked before launch and reported in Jeong et al. (2013). Same value of angular resolution was obtained in space, which prooves that telescope was not damaged during launch. 


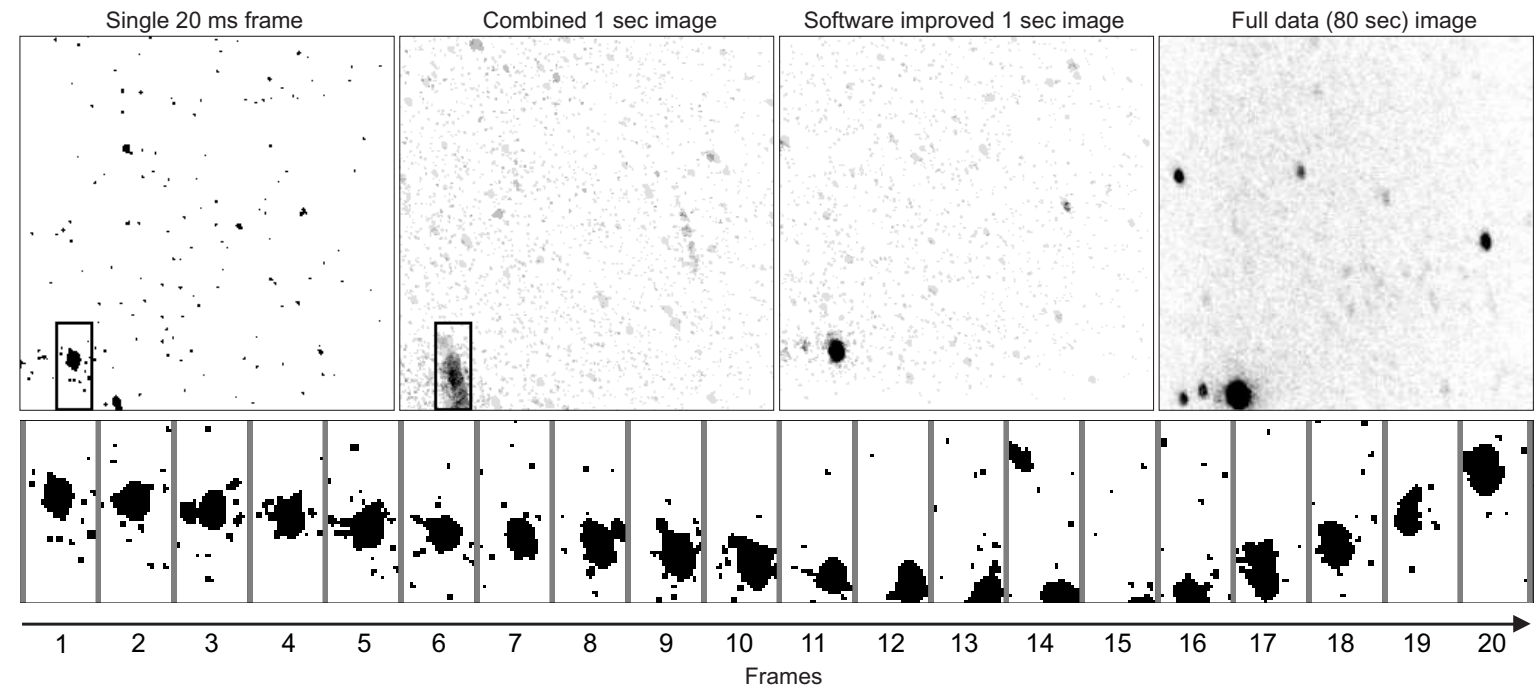

Figure 3: First: single 1-bit 20 ms exposure frame. Bright star (Tycho-2 1206-461-1) is selected to illustrate drift of FoV. Second: 20 frames are combined without drift compensation - image blurred, track of bright star may be observed. Third: same frames combined with full drift compensation - resolution became better - beside a bright star, two dim stars become observable. Fourth: All data (1700 frames, 80 seconds observation time) combined. Bottom: Movement of the bright star on over 20 frames. Frames 1-15: star is drifting down due to satellite rotation. Frames 16-20: correction of telescope FoV by slewing mirror, the star is returning back. Frame 20: The mirror drift compensation finish; star is returned to original position with $\sim 1^{\prime}$ mistake

We present part of our data on Fig. 4, which was taken on September 26 during imaging test. Image has original exposure 1 second. Slightly different exposures and different ICCD efficiencies of each pixel are taken into account (image is normalized). The telescope got enough photons to observe stars with B magnitudes up to 15.5; red magnitudes up to 14.5. Star ID are taken from Tycho-2 catalog. For magnitude check, USNO-B1.0 catalog B2 magnitudes were taken (in yellow), and R2 magnitudes (in red).
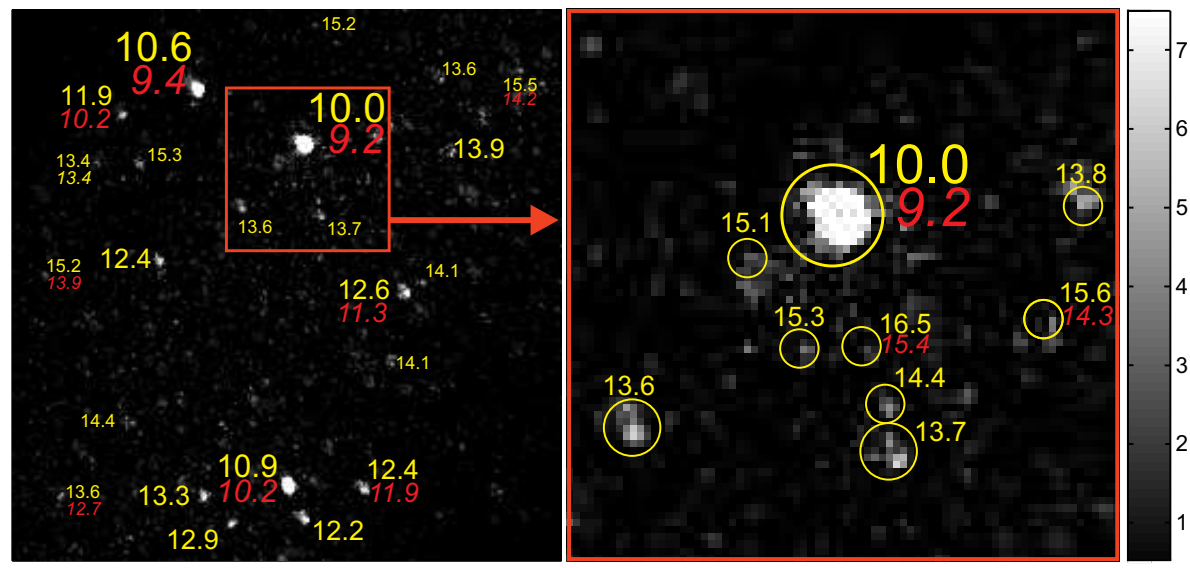

Figure 4: Example of SMT space data. Yellow - magnitude B2 from USNO-B1.0 catalogue. Red and italic - magnitude R2. Data is taken from July 25 UFFO test. 


\section{Conclusions}

The concept of slewing mirror telescope has been proved in space. Our space tests showed, that SMT has 1.4 seconds response time and is able to track astrophysical objects for more than 150 seconds (despite the happened problem with mirror position reading system).

The Slewing Mirror Telescope is the first telescope, utilizing a motorized mirror for changing optical beam path. The work of this concept is proved now by successful performance of SMT in space. From now on, the slewing mirror approach may be used in other space missions. It becomes especially useful in spacecrafts with multiple payloads, like Lomonosov, where the rotation of the whole satellite is not allowed because it changes the field of view of other payloads. Typical examples are interplanetary missions - they always have many payloads, and must keep orientation, with antenna looking towards Earth.

After launch, SMT got a problem with one of sensors of mirror position. This problem is overcome by especial software option, which enables mirror to move without feedback. After the problem overcome, the SMT has been calibrated by the in-situ method. As for now, the UFFOpathfinder is operating in orbit and the SMT is ready to observe the early part of optical and UV emission from GRBs. SMT is able to shed light on GRB prompt emission, never systematically studied before by other observatories. The results of GRB detection by the SMT will be reported in our following papers.

\section{References}

R. Willingale and P. Meszaros. Gamma ray bursts and fast transients multi-wavelength observations and multi-messenger signals. Space Sci Rev, 2017.

V. Connaughton, E. Burns, A. Goldstein, et al. Fermi gbm observations of ligo gravitational wave event gw150914. ApJl, 2016. doi: 10.3847/2041-8205/826/1/L6.

N. Gehrels, G . Chincarini, P. Giommi, et al. The swift gamma-ray burst mission. ApJ, 611(2): 1005-1020, 2004.

I. H. Park, B. Grossan, H. Lim, et al. The uffo (ultra-fast flash observatory) pathfinder. 2012.

I.H. Park, I.H. Panasyuk, V. Reglero, et al. Uffo/lomonosov : The payload for the observation of early photons from gamma ray bursts. 2017.

S. Jeong, J. W. Nam, K. B. Ahn, et al. Slewing mirror telescope optics for the early observation of uv/optical photons from gamma-ray bursts. Opt. Express, 21(2):2263-2278, 2013.

J. E. Kim, H. Lim, J. W. Nam, et al. Readout of the uffo slewing mirror telescope to detect uv/optical photons from gamma-ray bursts. JINST, 2013.

S. Jeong, M. I. Panasyuk, V. Reglero, et al. Ubat of uffo/lomonosov: The x-ray spacetelescope to observe early photons from gamma-ray bursts. Space Sci. Rev., 2017. 